\title{
Total energy density as an interpretative tool
}

\author{
Morrel H. Cohen \\ Department of Physics and Astronomy, Rutgers University, 136 Frelinghausen Road, Piscataway, NJ 08854 -8019 \\ Derek Frydel \\ Department of Chemistry, Rutgers University, 315 Penn Street, Camden, NJ 08102 \\ Kieron Burke \\ Department of Chemistry, Rutgers University, 610 Taylor Road, Piscataway, NJ 08854 \\ Eberhard Engel \\ Institut für Theoretische Physik, J.W. Goethe-Universität Frankfurt, Robert-Mayer-Strasse 8-10, D-60054, Frankfurt am \\ Main, Germany
}

(In preparation for J. Chem. Phys, August 10, 2018)

\begin{abstract}
We present an unambiguous formulation for the total energy density within density-functional theory. We propose that it be used as a tool for the interpretation of computed energy and electronic structure changes during structural transformations and chemical reactions, augmenting the present use of electron density changes and changes in the Kohn-Sham local density of states and Kohn-Sham energy density.
\end{abstract}

Changes in the electron density of a system undergoing a structural transformation or a chemical reaction have long been used to understand the driving forces underlying the transformation or the reaction. Indeed, the electron density itself has been regarded as one of the most significant descriptors of a system of electrons and nuclei since the advent of quantum mechanics. This view of the importance of the electron density was greatly strengthened by the emergence of density-functional theory. The Hohenberg-Kohn theorem [1] showed that the total energy of a system could be treated as a functional of the electron density. The Kohn-Sham theory [2] makes possible the construction of the true groundstate electron density of an $N$-electron system from the $N$ one-electron orbitals of lowest energy of a particular independent-electron system. This rigorous decomposition of the electron density into contributions from one-electron orbitals, the Kohn-Sham orbitals, greatly increases its power as an interpretive tool.

At present, methods based on density-functional theory are dominant in first-principles calculations for condensed matter and for much of quantum chemistry. The electron density and its Kohn-Sham orbital decomposition, fundamental outputs of the computations, are typically used to enrich understanding of the resulting energetics. For example, studies of the isosurfaces of electron density changes along a reaction pathway for the dissociation of $\mathrm{H}_{2}$ on the $\mathrm{Pd}$ (100) surface [3] have revealed the formation of bridge bonds between the s-p tails of the metallic surface electron density and the $\sigma_{g}$ and $\sigma_{u}^{*}$ molecular orbitals. These bridge bonds mediate the hybridization of the molecular orbitals with the metallic d-orbitals before they actually overlap. It is the bridge bonds which evolve into the bonds between the dissoci- ated hydrogen atoms and the metal.

The Kohn-Sham theory enables one to go still deeper into the interpretation of the results of the electronicstructure computations. For extended systems, one can construct the Kohn-Sham density of states,

$$
g(\epsilon)=\sum_{i=1}^{N} \delta\left(\epsilon-\epsilon_{i}\right),
$$

where $\epsilon_{i}$ is one of the $N$ lowest Kohn-Sham eigenvalues, and use it to tease out the energetics associated with the electron-density changes. For example, in studies of the interaction of chemisorbed $\mathrm{O}$ and $\mathrm{H}$ to form $\mathrm{OH}$ and $\mathrm{H}_{2} \mathrm{O}$ on the (111) surfaces of $\mathrm{Rh}$ and $\mathrm{Pt}$ 顿, the resonances and bound states associated with the bonding and antibonding orbitals of the atoms, radicals, or molecules to the surface are shown to be clearly visible in $g(\epsilon)$. Even more revealing is the local density of states,

$$
g(\mathbf{r}, \epsilon)=\sum_{i=1}^{N}\left|\phi_{i}(\mathbf{r})\right|^{2} \delta\left(\epsilon-\epsilon_{i}\right),
$$

where $\phi_{i}(\mathbf{r})$ is one of the occupied Kohn-Sham orbitals, which enables one to associate a particular resonance or bound state in the Kohn-Sham spectrum, Eq.(11), with a particular atom or molecule. It allows one to make a resolution with respect to the Kohn-Sham energy of the changes of electron density accompanying structural transitions or chemical reactions.

Nevertheless, what drives a transformation or a reaction is the dependence of the total energy on the nuclear coordinates. Thus, what one needs as an additional interpretive tool is an unambiguous, computationally feasible total-energy density $e(\mathbf{r})$, such that 


$$
E=\int d^{3} r e(\mathbf{r})
$$

where $E$ is the total energy. With $e(\mathbf{r})$ one could explore the relation between local changes in the electron density and the spatial dispersion of the total energy changes through the corresponding changes in $e(\mathbf{r})$.

To be more specific, in Ref. [3], evidence was found for the simultaneous presence of all the specific mechanisms commonly cited as playing important roles in the dissociation of $\mathrm{H}_{2}$ on a transition metal surface. These included the formation of an orthogonality hole in the $s-p$ tail of the metallic electron density, with concommitant flow of screening charge into the $d$-states, as emphasized by Harris and Andersson [5]; the hybridization of the $d$ states with the occupied $\sigma_{g}$ bonding orbital of the $\mathrm{H}_{2}$, as emphasized by Hammer and Scheffler [6], and the hybridization of the unoccupied $\sigma_{u}^{*}$ antibonding orbital of the $\mathrm{H}_{2}$ with associated backbonding, as emphasized by Hammer and Nørskov [7,8]. Each of these processes introduced distinct, easily recognized characteristic changes in the electron density, which occured in distinct regions of space. One could therefore utilize $e(\mathbf{r})$ to order the relative importance of each of these mechanisms as well as of bridge-bond formation at different locations along the dissociation pathway, garnering thereby a detailed, intimate quantitative understanding of the breaking of the intramolecular bond and the formation of metal-atom bonds in the course of the dissociation.

There are several difficulties to be surmounted in constructing an unambiguous, computationally-feasible expression for $e(\mathbf{r})$, as will be discussed below. To avoid the difficulties, only the Kohn-Sham energy density $e_{\mathrm{S}}(\mathbf{r})$,

$$
e_{\mathrm{S}}(\mathbf{r})=\sum_{i=1}^{N} \epsilon_{i}\left|\phi_{i}(\mathbf{r})\right|^{2}=\int^{\epsilon_{N}^{+}} d \epsilon g(\mathbf{r}, \epsilon)
$$

was used as an interpretive tool in reference [4], though to good effect. In the present paper, we present an explicit formulation for $e(\mathbf{r})$ which is both unambiguous and computationally feasible, as required.

The total energy can be represented as a density functional in the form

$$
E=T+V_{\text {ee }}+V_{e n}+V_{n n}
$$

where $T, V_{\text {ee }}$, and $V_{\text {en }}$ are the functionals for the electron kinetic energy, the electron-electron interaction, and the electron-nuclear interaction. $V_{n n}$ is the Coulomb interaction between the nuclei. In Kohn-Sham densityfunctional theory [9, 10], the expression Eq. (5) is rewritten as

$$
E=T_{\mathrm{S}}+U+E_{\mathrm{xC}}+V_{e n}+V_{n n}
$$

Here $T_{\mathrm{S}}$ is the kinetic energy of the independent KohnSham particles, $\mathrm{U}$ is the Hartree electrostatic energy,

$$
U=\frac{1}{2} \int d^{3} r \int d^{3} r^{\prime} \frac{n(\mathbf{r}) n\left(\mathbf{r}^{\prime}\right)}{\left|\mathbf{r}-\mathbf{r}^{\prime}\right|},
$$

where $n(\mathbf{r})$ is the electron density, and $E_{\mathrm{xC}}$ is the exchange-correlation energy,

$$
E_{\mathrm{xC}}=T-T_{\mathrm{S}}+V_{\mathrm{ee}}-U
$$

On the other hand, the total Kohn-Sham energy of the system is

$$
E_{\mathrm{S}}=\sum_{i=1}^{N} \epsilon_{i}=T_{\mathrm{S}}+\int d^{3} r n(\mathbf{r}) v_{\mathrm{S}}(\mathbf{r}),
$$

In Eq.(9), $v_{\mathrm{S}}(\mathbf{r})$ is the Kohn-Sham potential,

$$
v_{\mathrm{S}}(\mathbf{r})=v_{e n}(\mathbf{r})+v_{\mathrm{H}}(\mathbf{r})+v_{\mathrm{XC}}(\mathbf{r}),
$$

where $v_{e n}(\mathbf{r})$ is the Coulomb potential produced by the nuclei at $\mathbf{r}$, which enters $V_{\text {en }}$ as well

$$
V_{e n}=\int d^{3} r n(\mathbf{r}) v_{e n}(\mathbf{r}),
$$

$v_{\mathrm{H}}(\mathbf{r})$ is the Hartree potential, the mean electrostatic potential produced by the electrons,

$$
v_{\mathrm{H}}(\mathbf{r})=\int d^{3} r^{\prime} \frac{1}{\left|\mathbf{r}-\mathbf{r}^{\prime}\right|} n\left(\mathbf{r}^{\prime}\right),
$$

which enters the Hartree energy functional,

$$
U=\int d^{3} r e_{\mathrm{H}}(\mathbf{r}), \quad e_{\mathrm{H}}(\mathbf{r})=\frac{1}{2} n(\mathbf{r}) v_{\mathrm{H}}(\mathbf{r}) .
$$

Finally, $v_{\mathrm{XC}}(\mathbf{r})$ is the functional derivative of $E_{\mathrm{XC}}$ :

$$
v_{\mathrm{XC}}(\mathbf{r})=\frac{\delta E_{\mathrm{XC}}}{\delta n(\mathbf{r})}
$$

Thus Eq.(9) can be transformed into

$$
E_{\mathrm{S}}=T_{\mathrm{S}}+2 U+V_{e n}+\int d^{3} r n(\mathbf{r}) v_{\mathrm{XC}}(\mathbf{r}) .
$$

Inserting Eq.(15) into Eq.(5) allows us to eliminate $T_{\mathrm{S}}$, resulting in

$$
E=E_{\mathrm{S}}-U+V_{n n}+E_{\mathrm{XC}}-\int d^{3} r n(\mathbf{r}) v_{\mathrm{XC}}(\mathbf{r}) .
$$

Each of the above terms can unambiguously be written as an integral over an energy density so that

$$
\begin{aligned}
e(\mathbf{r}) & =e_{\mathrm{S}}(\mathbf{r})-e_{\mathrm{H}}(\mathbf{r})-\frac{1}{2} \rho_{n}(\mathbf{r}) v_{e n}(\mathbf{r}) \\
& +e_{\mathrm{XC}}(\mathbf{r})-n(\mathbf{r}) v_{\mathrm{XC}}(\mathbf{r}) .
\end{aligned}
$$

In this expression, $e_{\mathrm{S}}(\mathbf{r})$ is given by Eq.(4) and can readily be constructed from the output of the standard codes 
which solve the Kohn-Sham equations. The electron density $n(\mathbf{r})$ is a fundamental output of such computations. The potentials $v_{\mathrm{H}}(\mathbf{r})$ and $v_{e n}(\mathbf{r})$ must be constructed during the computations, as must be $v_{\mathrm{XC}}(\mathbf{r})$. The number density of the nuclei weighted by their respective atomic numbers is

$$
\rho_{n}(\mathbf{r})=\sum_{\alpha} Z_{\alpha} \delta\left(\mathbf{r}-\mathbf{R}_{\alpha}\right)
$$

where $\mathbf{R}_{\alpha}$ is the position and $Z_{\alpha}$ the charge of nucleus $\alpha$. We note that the third term in Eq.(17), though singular at the nuclei, vanishes between the nuclei.

The exchange-correlation energy density $e_{\mathrm{xC}}(\mathbf{r})$ is defined in terms of $v_{\mathrm{Xc}}[n](\mathbf{r})$ via the procedure of Burke, Cruz, and Lam (BCL) [11], an extension of the original ideas of Engel and Vosko [12],

$$
\nabla^{2} e_{\mathrm{XC}}(\mathbf{r})=3 \nabla\left(n(\mathbf{r}) \nabla \tilde{v}_{\mathrm{XC}}(\mathbf{r})\right)
$$

where

$$
\tilde{v}_{\mathrm{XC}}[n](\mathbf{r})=\int_{0}^{1} \frac{d \gamma}{\gamma} v_{\mathrm{XC}}\left[n_{\gamma}\right]\left(\frac{\mathbf{r}}{\gamma}\right)
$$

and $n_{\gamma}(\mathbf{r})=\gamma^{3} n(\gamma \mathbf{r})$. Their definition has several advantages in this context. Most importantly, the BCL procedure defines an exchange-correlation energy density unambiguously in terms of any given exchange-correlation energy functional (including the exact one), since it uses the potential. Thus exchange-correlation energy densities calculated this way are approximations to an exact quantity. Within the local density approximation (LDA), their procedure reproduces simply $e_{\mathrm{XC}}^{\text {unif }}(n(\mathbf{r}))$, the exchange-correlation energy density of a uniform electron gas of density $n(\mathbf{r})$. This is just the conventional energy density within LDA, and so is already calculated in standard LDA Kohn-Sham calculations. Within any generalized gradient approximation (GGA) such as PBE [13 or BLYP 114,15, their procedure defines a different energy density from the conventional one. This energy density includes dependencies on the Laplacian and other higher derivatives of $n(\mathbf{r})$, and so is far more sensitive to details in $n(\mathbf{r})$ than the conventional GGA forms. The scaling defined in Eq. (20) is easily performed on any approximate density functional. Finally, the BCL procedure defines an exact unambiguous exchange energy density, so that it can be applied even to hybrids of GGA with exact exchange [11].

To illustrate this energy density, we plot the various contributions to the total energy density for the Na atom. Our calculations are all for exact exchange only, using the atomic optimized effective potential (OEP) code of Engel 16. From Figs. 1 and 2, we see that the total energy density is usually dominated by two terms: the Kohn-Sham eigenvalue contribution and the electrostatic energy correction. The two exchange terms of Eq. (17)

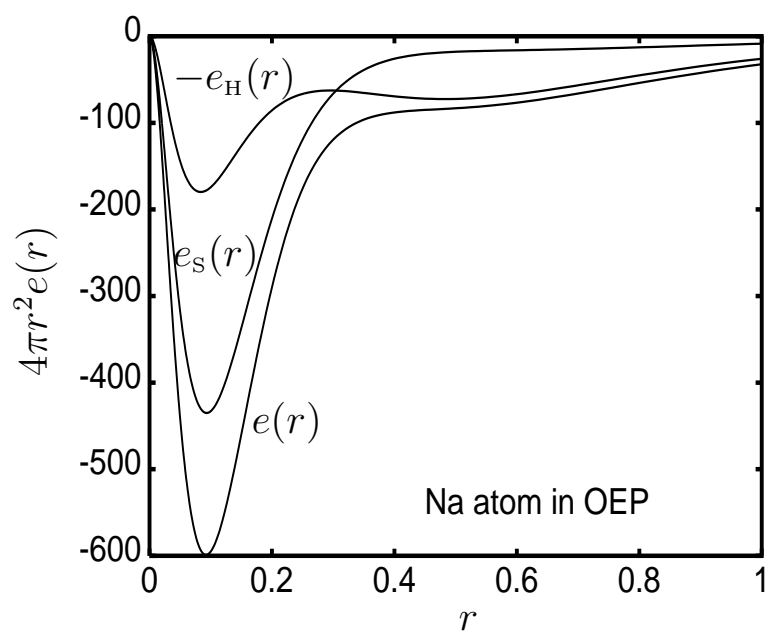

FIG. 1. Radial contributions to the total electronic energy density for the $\mathrm{Na}$ atom (atomic units).

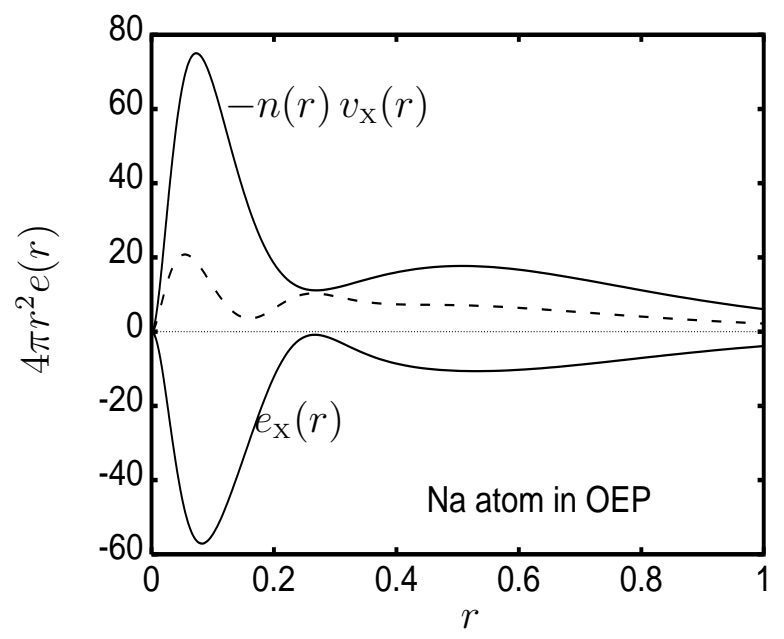

FIG. 2. Radial exchange contributions to the total electronic energy density for the $\mathrm{Na}$ atom (atomic units); the dashed curve is the sum of the other two. 


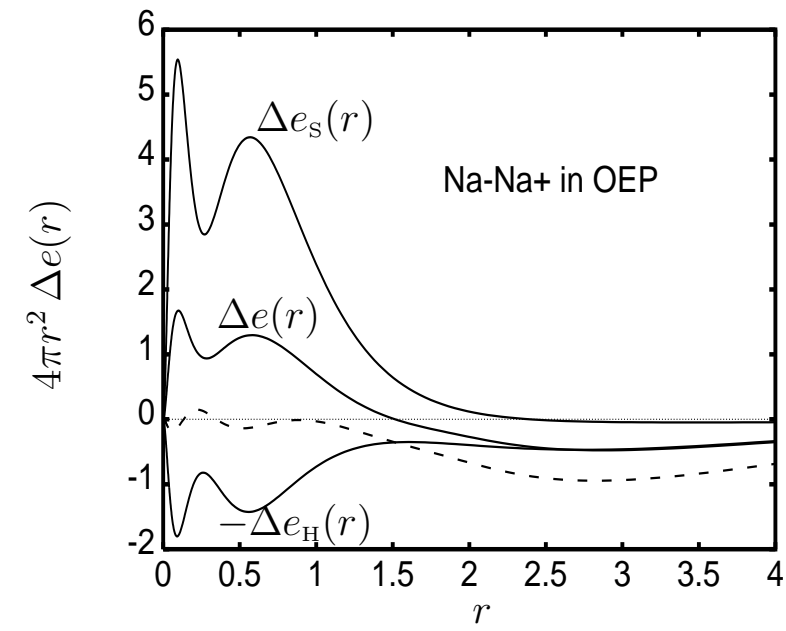

FIG. 3. Same as Fig. 1, but for the difference between the neutral atom, $\mathrm{Na}$, and the ion, $\mathrm{Na}^{+}$; the dashed curve is $\Delta e(r)-\Delta e_{\mathrm{H}}(r)$.

are small, and largely cancel, as shown in Fig. 2. This cancellation is already apparent in LDA exchange, where the sum of these terms is only $1 / 3$ of $e_{\mathrm{x}}^{\text {unif }}$. This agrees with arguments 17 19 that changes in the energy can be largely understood in terms of changes in KS eigenvalues and the electrostatic potentials. The present analysis opens the possibility of extending these arguments to the exact functional.

To illustrate the importance of the electrostatic energy correction, consider Fig. 3, which shows energy density differences between neutral $\mathrm{Na}$ and its ion. The KohnSham contribution is dominated by the core $(r \leq 2$, roughly). This is because the 3 s electron in $\mathrm{Na}$ induces an almost constant shift in the core Hartree potential, so that all eigenvalues are about 0.29 higher in the neutral relative to the ion. When the Hartree correction is made, and the total energy density difference plotted, we find that there are two almost equal and opposite contributions to the energy difference. To see that these are entirely electrostatic effects, we further subtract $e_{\mathrm{H}}(r)$ from the total (dashed line), showing that, in the absence of Hartree contributions, the total energy density lies almost entirely in the valence region. This simple analysis demonstrates both the importance of electrostatic contributions to the energy density, and how the various terms are needed in Eq. (17) to produce the final physical picture. (For example, overall shifts in KS eigenvalues do not contribute to $e(\mathbf{r})$, but do show up in $e_{\mathrm{S}}(\mathbf{r})$.) The electrostatic energy correction is omitted in the generalized perturbation method [20] widely used in the theory of alloys 21. The present analysis allows us to explore the role of exhange-correlation energy differences in chemical systems.

Note that $v_{\mathrm{H}}(\mathbf{r})$ and $v_{e n}(\mathbf{r})$ each diverge separately in the thermodynamic limit for extended systems. The divergences cancel each other in $v_{\mathrm{S}}(\mathbf{r})$, Eq.(10), because only their sum enters. The divergences similarly cancel in $E$, Eqs.(5), (6), and (16) and in $E_{\mathrm{S}}$, Eq.(9). They do not cancel in $e(\mathbf{r})$ as written in Eq.(17). This difficulty should be obviated by the methods of eliminating the singular parts of $v_{\mathrm{H}}(\mathbf{r})$ and $v_{e n}(\mathbf{r})$ which are standard in the theory of the electron structure of extended systems. That is, $v_{\mathrm{H}}(\mathbf{r})$ and $v_{e n}(\mathbf{r})$ should be interpreted as the nonsingular part of the Hartree and nuclear potential, respectively, in Eq.(17) for extended systems.

In conclusion, we propose (1.) that the total energy density between two nuclei be constructed from the output of Kohn-Sham computations augmented by the procedure of ref. [11] and used as an interpretive tool in analyzing energy and electronic structure changes during transformations and reactions when the focus is on the study of bond formation in the spaces between atoms. We propose in addition (2.) that the full expression for $e(\mathbf{r}), \mathrm{Eq} .(17)$, be coarse-grained by integration over a small sphere containing a particular nucleus to examine the role of the corresponding atom in chemical bonding or structural transformation. Such spheres are already present in, e.g., linear-augmented-plane-wave, muffin-tin, and KKR codes and can be readily introduced into other procedures.

\section{ACKNOWLEDGMENT}

This work was initiated at the Aspen center of Physics and carried forward at the Department of Physics of Rutgers University (New Brunswick). D.F. and K.B. were supported by a grant from Research Corporation, and the National Science Foundation under grant number We thank Eberhard Engel for the use of his atomic OEP code.

[1] Inhomogeneous electron gas, P. Hohenberg and W. Kohn, Phys. Rev. 136, B 864 (1964).

[2] Self-consistent equations including exchange and correlation effects, W. Kohn and L.J. Sham, Phys. Rev. 140, A 1133 (1965).

[3] Mechanisms in the dissociation of $\mathrm{H}_{2}$ on Pd (100), S. Wilke, V. Natoli, M. H. Cohen, in preparation.

[4] Theoretical investigation of water formation on $R h$ and Pt surfaces, S. Wilke, V. Natoli, M. H. Cohen, in preparation.

[5] $\mathrm{H}_{2}$ dissociation at metal surfaces, J. Harris and S. Andersson, Phys. Rev. Lett. 55, 1583 (1985).

[6] Local Chemical Reactivity of a Metal Alloy Surface, B. Hammer and M. Scheffler, Phys. Rev. Lett. 74, 3487 (1995).

[7] Why gold is the noblest of all the metals, B. Hammer and J.K. Nørskov, Nature 376, 238 (1995). 
[8] B. Hammer and J.K. Nørskov, Surf. Sci. 343, 211 (1995).

[9] R.M. Dreizler and E.K.U. Gross, Density Functional Theory (Springer-Verlag, Berlin, 1990).

[10] Density Functional Theory of Atoms and Molecules, R.G. Parr and W. Yang (Oxford, New York, 1989).

[11] Unambiguous exchange-correlation energy density, K. Burke, F.G. Cruz, and K.C. Lam, J. Chem. Phys. 109, 8161 (1998).

[12] Exact exchange-only potentials and the virial relation as microscopic criteria for generalized gradient approximations, E. Engel and S.H. Vosko, Phys. Rev. B 47, 13164 (1993).

[13] Generalized gradient approximation made simple, J.P. Perdew, K. Burke, and M. Ernzerhof, Phys. Rev. Lett. 77, 3865 (1996); 78, 1396 (1997) (E).

[14] Density-functional exchange-energy approximation with correct asymptotic behavior, A.D. Becke, Phys. Rev. A 38, 3098 (1988).

[15] Development of the Colle-Salvetti correlation-energy formula into a functional of the electron density, C. Lee, W. Yang, and R.G. Parr, Phys. Rev. B 37, 785 (1988).

[16] From explicit to implicit density functionals, E. Engel and R.M. Dreizler, J. Comput. Chem. 20, 31 (1999).

[17] Individual orbital contributions to the SCF virial in homonuclear diatomic molecules, D.G. Pettifor, J. Chem. Phys. 69, 2930 (1978).

[18] The electronic structure of transition metals, A.R. Mackintosh and O.K. Andersen, in Electrons at the Fermi Surface, ed. M. Springford, (Cambridge University Press, Cambridge, 1980).

[19] Theory of adsorption and surface reactions, B. Hammer and J.K. Nörskov, in Chemisorption and reactivity on supported clusters and thin films, eds. R.M. Lambert and G. Pacchioni (Kluwer, Holland, 1997).

[20] Generalized perturbation theory in disordered transitional alloys-applications to calculation of ordering energies, $\mathrm{F}$. Ducastelle and F. Gautier, J. Phys. F 6, 2039 (1976).

[21] First principles approaches to surface segregation, R. Monnier, Phil. Mag. B, 75, 67 (1997). 